\title{
Editorial
}

\section{Nonlinear Elliptic Systems and Nonlinear Parabolic Systems}

\author{
Li Wei, ${ }^{1}$ Anthony To-Ming Lau, ${ }^{2}$ Hongya Gao, ${ }^{3}$ and Zhongbo Fang ${ }^{4}$ \\ ${ }^{1}$ School of Mathematics and Statistics, Hebei University of Economics and Business, Shijiazhuang 050061, China \\ ${ }^{2}$ Department of Mathematical and Statistical Sciences, University of Alberta, Edmonton, AB, Canada T6G 2 G1 \\ ${ }^{3}$ College of Mathematics and Computer Science, Hebei University, Baoding 071002, China \\ ${ }^{4}$ School of Mathematical Sciences, Ocean University of China, Qingdao 266100, China
}

Correspondence should be addressed to Li Wei; diandianba@yahoo.com

Received 10 July 2014; Accepted 10 July 2014; Published 16 July 2014

Copyright (C) $2014 \mathrm{Li}$ Wei et al. This is an open access article distributed under the Creative Commons Attribution License, which permits unrestricted use, distribution, and reproduction in any medium, provided the original work is properly cited.

Nonlinear elliptic and parabolic differential equations play important roles in applied mathematics since they can describe many phenomena arising in mathematical physics, engineering fields, electricity, fluid dynamics, and many other fields, such as the filtration theory, phase inversion theory, biochemistry, the dynamics of biology, and non-Newtonian theory. Hence, it is important to develop novel theories and methods to investigate nonlinear elliptic or parabolic systems or related topics. The papers selected for this special issue represent a good panel for addressing this challenge. Of course, the selected topic and the papers are not an exhaustive representation of the area of nonlinear elliptic systems and nonlinear parabolic systems. Nonetheless, they represent the rich and many-facet knowledge, which we have the pleasure of sharing with the readers.

The special issue contains nine papers, where two papers are related to $A$-harmonic equation. One paper introduces a new method of 3D facial expression animation. One paper is regarding the Li-Yorke sensitivity of set-valued discrete systems and one paper is considering inverse estimates for nonhomogeneous backward heat problem. Finally, four papers cover the iterative algorithms for split feasibility problem, equilibrium problem, variational inequalities, and so forth.

In a paper entitled "Higher integrability for very weak solutions of inhomogeneous A-harmonic form equations," Y. Tong et al. study an $A$-harmonic form equation with more general growth conditions than the usual ones. The higher integrability for very weak solutions is proved.

In a paper entitled "Inverse estimates for nonhomogeneous backward heat problems," T. Min et al. investigate the inverse problem in the nonhomogeneous heat equation involving the recovery of the initial temperature from measurements of the final temperature. This problem is known as the backward heat problem and is severely ill-posed. They show that this problem can be converted into the first Fredholm integral equation and an algorithm of inversion is given using Tikhonov's regularization method. The genetic algorithm for obtaining the regularization parameter is presented. They also present numerical computations that verify the accuracy of their approximation.

In the paper entitled "New mixed equilibrium problems and iterative algorithms for fixed point problems in Banach spaces," M. Chen et al. first introduce a new mixed equilibrium problem with a relaxed monotone mapping in Banach spaces and then prove the existence of solutions of the equilibrium problem. Later, they introduce a new iterative algorithm for finding a common element of the set of solutions of the equilibrium problem and the set of fixed points of a quasi- $\varphi$-nonexpansive mapping and prove some strong convergence theorems of the iteration.

In the paper entitled "Li-Yorke sensitivity of set-valued discrete systems," $\mathrm{H}$. Liu et al. consider a surjective, continuous map $f: X \rightarrow X$ and a continuous map $\bar{f}$ of $\kappa(X)$ into itself introduced by $f$, where $X$ is a compact metric space and $\kappa(X)$ is the space of all nonempty compact subsets of $X$ endowed with the Hausdorff metric. They give a short proof of the fact that if $\bar{f}$ is Li-Yorke sensitive, then $f$ is Li-Yorke sensitive. They also give an example showing that the inverse is not always true; that is, if $f$ is Li-Yorke sensitive, then $\bar{f}$ may not be. 
In the paper entitled "Relaxed extragradient algorithms for the split feasibility problem," Y. Yu et al. introduce a new relaxed extragradient algorithm for split feasibility problem, which is widely used in signal processing and image reconstruction and so forth.

In the paper entitled "A new method of 3D facial expression animation," S. Sun and C. Ge introduce a novel expression ratio image (ERI) driving framework based on super vector regression (SVR) and MPEG-4 for automatic 3D facial expression animation. Through using the method of SVRM, the framework can learn and forecast the regression relationship between the facial animation parameters (FAP) and the parameters of expression ratio image. Firstly, they build a 3D face animation system derived by FAP. Secondly, using the method of principle component analysis (PCA), they generate the parameter sets of eigen-ERI space, which will rebuild reasonable expression ratio image. Then, they learn a model with the support vector regression mapping, and facial animation parameters can be synthesized quickly with parameters of eigen-ERI. Finally, they implement the 3D face animation system driving by the result of FAP.

In the paper entitled "Strong convergence of a modified Ishikawa iterative sequence for asymptotically quasi-pseudocontractive-type mappings," L. Li and Y. Wang investigate the strong convergence problem of a modified mixed Ishikawa iterative sequence with errors for approximating the fixed points of an asymptotically nonexpansive mapping in the intermediate sense and an asymptotically quasi-pseudocontractive-type mapping in an arbitrary real Banach space.

In the paper entitled "Convergence theorem for equilibrium and variational inequality problems and a family of infinitely nonexpansive mappings in Hilbert space," Z. Yinying et al. construct a hybrid iterative scheme for finding a common element of the set of common fixed points for a family of infinite nonexpansive mappings, the set of solutions of the variational inequality problem, and the equilibrium problem in Hilbert space. They prove some strong convergence theorems.

In the paper entitled "Zeros for the gradients of weakly A-harmonic tensors," Y. Tong et al. prove the Caccioppoli inequality of weakly $A$-harmonic tensors, which can be used to consider the weak reverse Holder inequality, regularity property, and zeros of weakly $A$-harmonic tensors.

\section{Acknowledgment}

We would like to thank the authors for their excellent contributions and patience in assisting us. Finally, the fundamental work of all the reviewers of these papers is also very warmly acknowledged. 


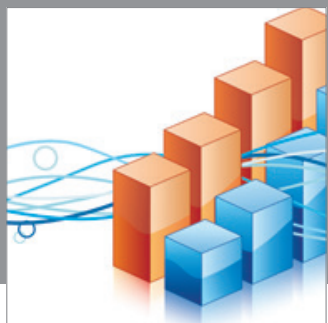

Advances in

Operations Research

mansans

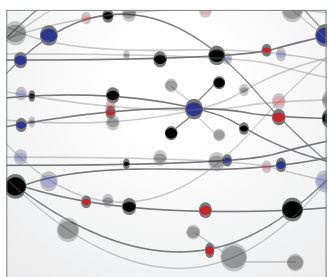

The Scientific World Journal
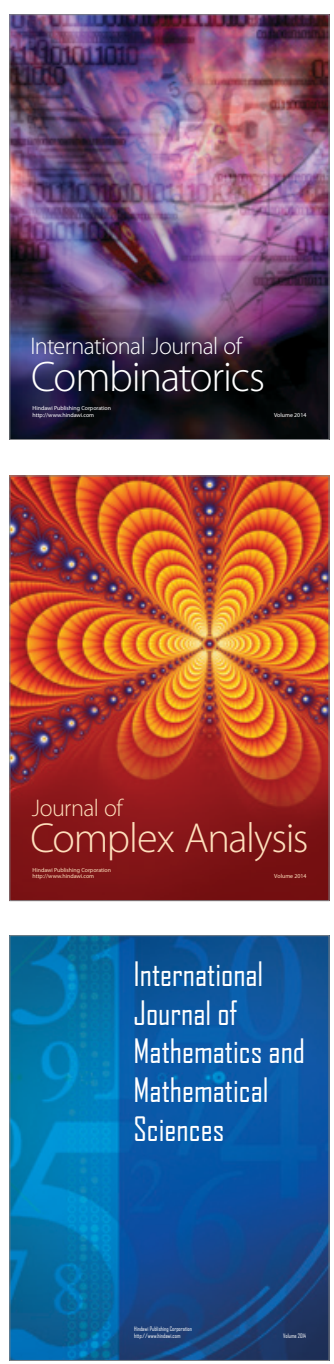
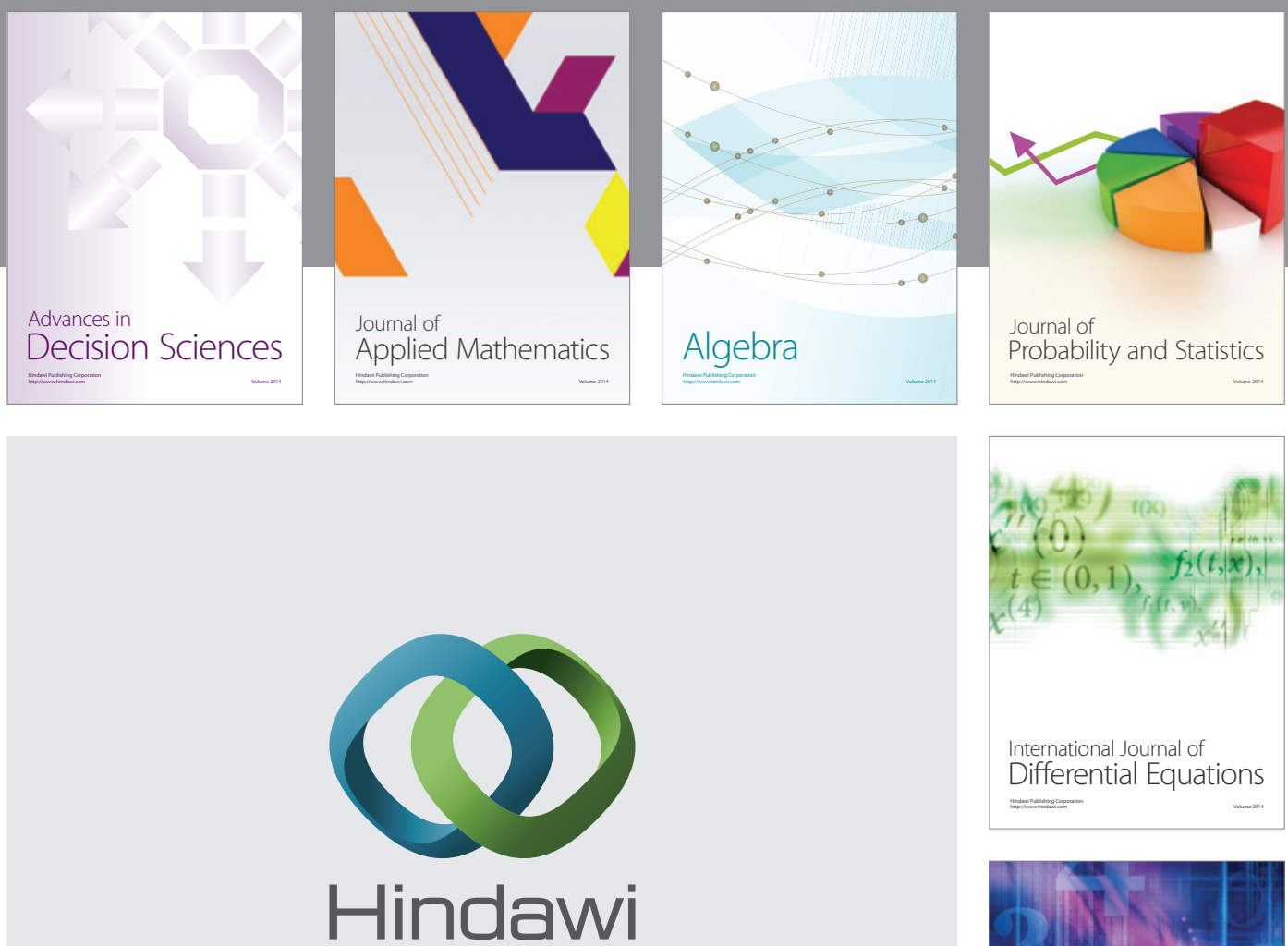

Submit your manuscripts at http://www.hindawi.com
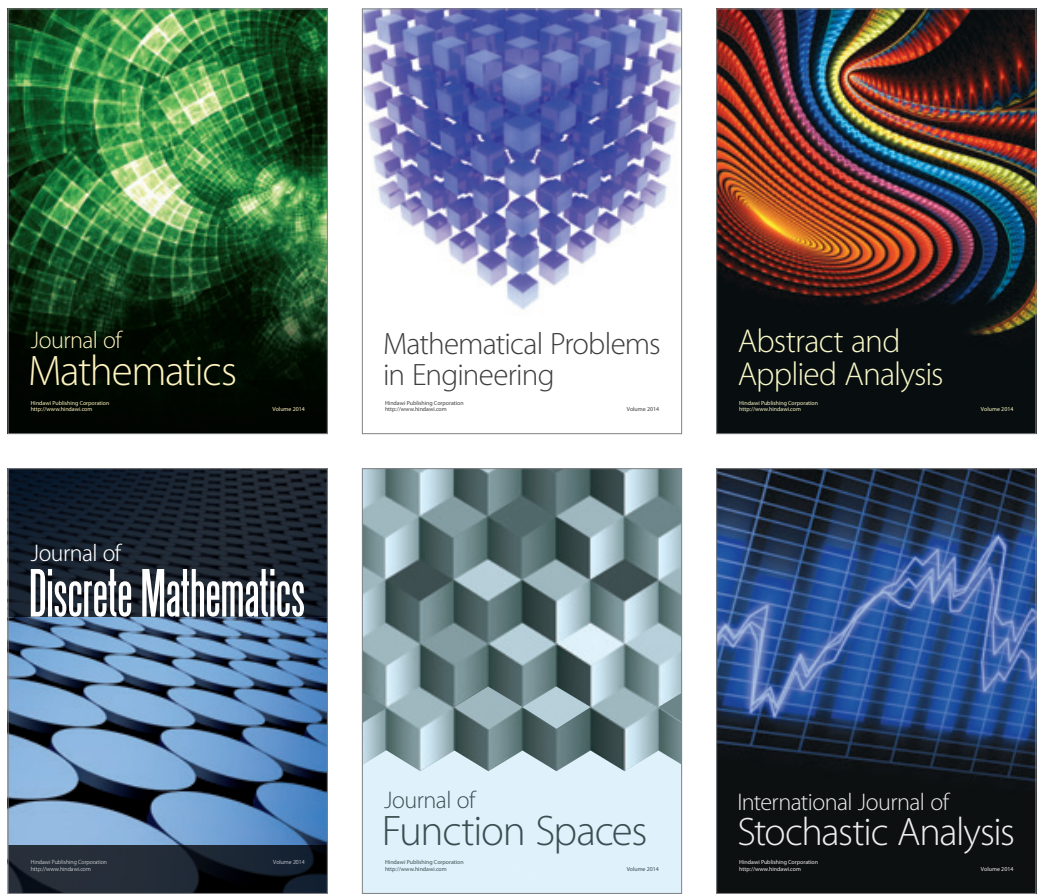

Journal of

Function Spaces

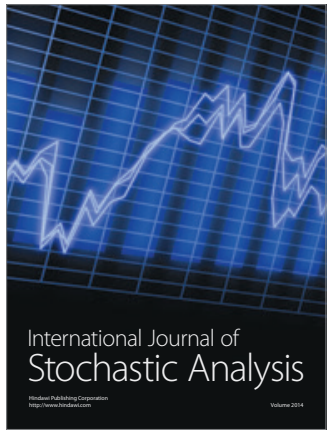

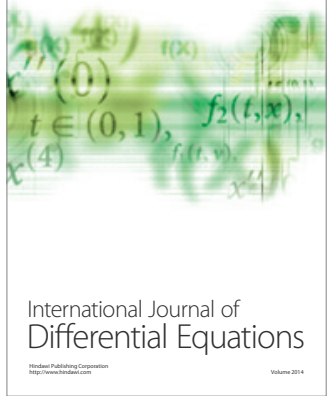
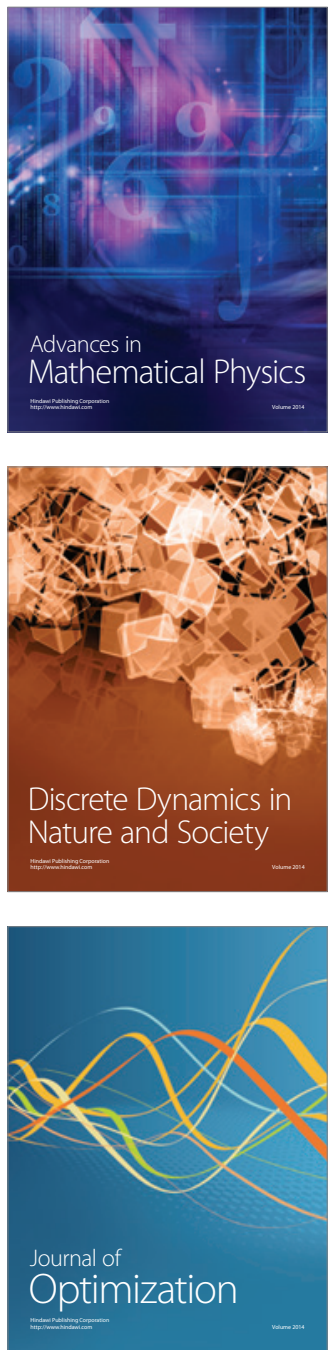